\title{
Simulation physique et numérique du comportement des fondations et ancrages en sols marins
}

\author{
Digital and physical simulation of the behaviour \\ of foundations and anchorings in marine soils
}

\author{
M. Boulon \\ Maître de Conférences, \\ IUT 1 de Grenoble
}

\author{
P. Foray
}

Maître de Conférences,

Ecole nationale supérieure

d'hydraulique de Grenoble

Le rapport concerne l'apport du laboratoire dans le domaine concerné. Il évoque :

- l'identification des mécanismes élémentaires mis en jeu dans le sol ou au contact sol-structure, utilisant les appareils classiques de laboratoire et les chambres de calibration; on simule dès ce stade la nature cyclique des sollicitations;

- la simulation numérique sur ordinateur, tenant compte des lentes modifications de propriétés mécaniques dues aux cycles, ainsi que de l'évolution des pressions interstitielles induites;

- enfin, la sélection des phénomènes majeurs, au vu des comparaisons entre simulations numériques et expériences, de manière à obtenir des méthodes de calcul prévisionnel fiables utilisables industriellement.

The report concerns the laboratory's contribution in the field in question. It covers :

- identification of elementary mechanisms installed in the soil or in contact with the soil-structure, using the conventional laboratory devices and calibration chambers. From this stage onwards, the cyclic nature of stresses are simulated;

- digital computer simulation, taking into account the slow modifications of mechanical properties due to cycles, as well as the evolution of induced interstitial pressures;

- finally, the selection of major phenomena, as regards the comparisons between digital simulations and experiences in order to obtain reliable estimated calculation methods which can be used industrially.

\section{Introduction}

Le comportement des fondations et ancrages dans les sols marins met en jeu un ensemble de phénomènes liés à :

- la complexité du type de fondations: les pieux terrestres eux-mêmes ont un comportement encore mal connu. En mer, les problèmes sont aggravés du fait de la taille des fondations (pieux de $1 \mathrm{~m}$ à $1,50 \mathrm{~m}$ de diamètre jusqu'à 100 mètres d'encastrement) et de leur mode de mise en place (battage depuis la surface avec des effets non maîtrisés sur le sol avoisinant);

- la complexité du mode de chargement : les efforts cycliques dûs à la houle et appliqués aux structures de plate-forme (ou de digues) se transmettent aux fondations et donc au sol entourant celles-ci. Les sédiments marins étant formés la plupart du temps d'éléments fins de faible perméabilité, le transfert des efforts répétés peut provoquer des sous pressions interstitielles qui diminuent la résistance mécanique du sol.

L'approche de ces problèmes à partir du laboratoire est complementaire de celle des essais in situ et vise deux objectifs : 


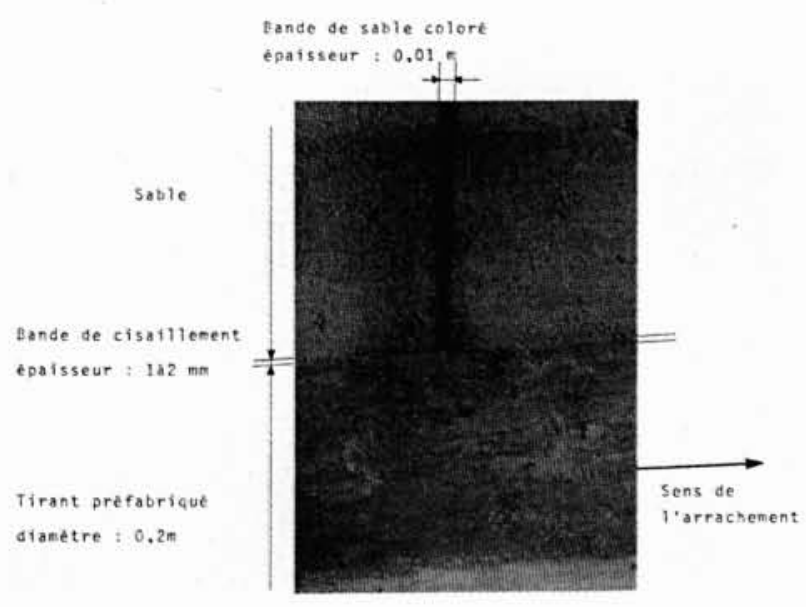

Figure 1. - d'après Plumelle [12].
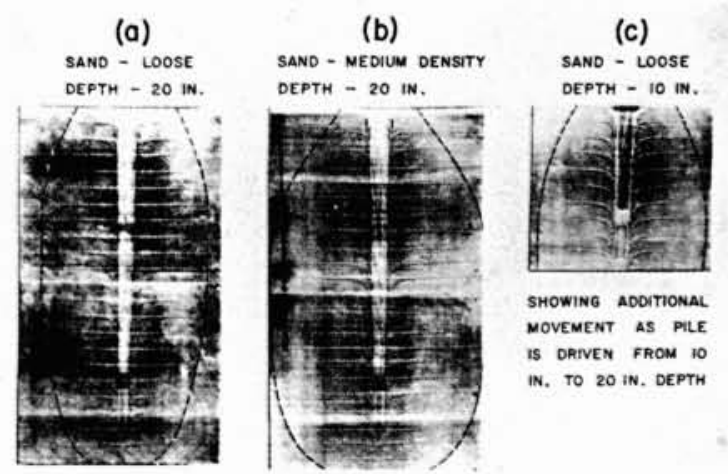

Figure 2. - d'après Robınsky et Morrisson [14].
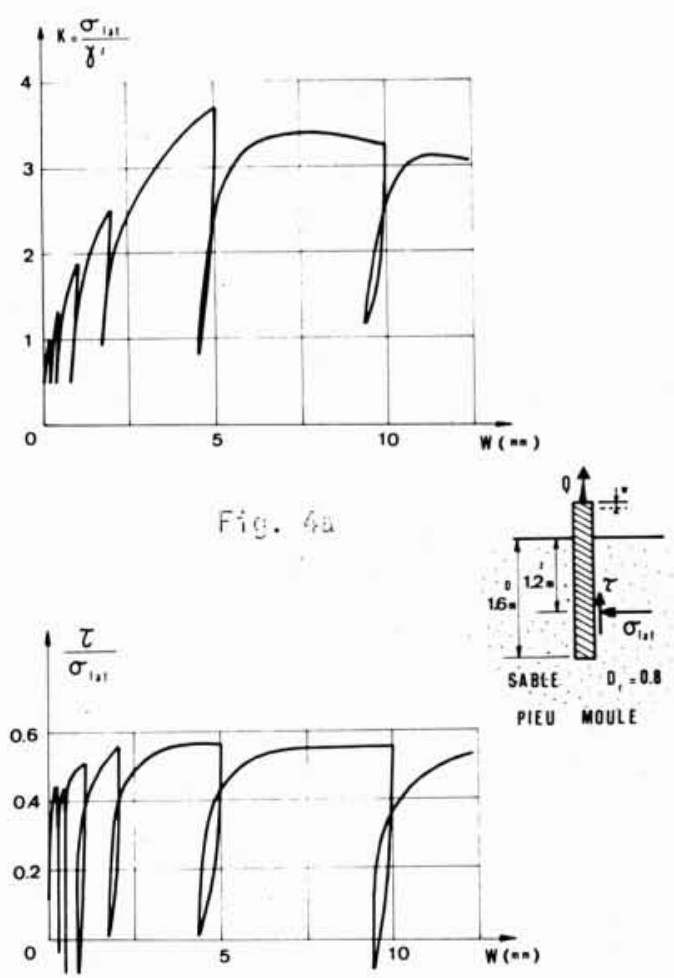

Figure 4 .

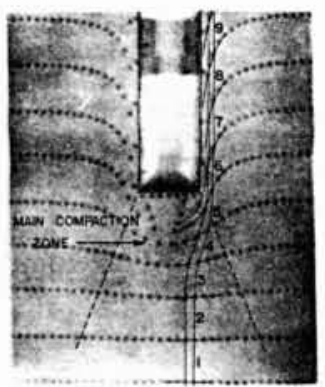

Figure 3. - daprès Robinsky et Morrisson [14].

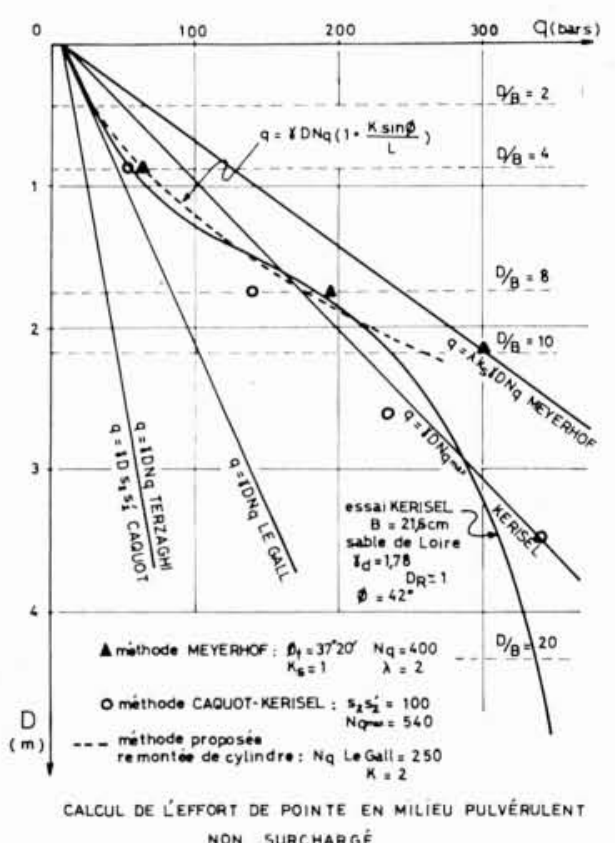

Figure 5. - Calcul de l'effort de poine en milieu pulvérulent non surchargé.

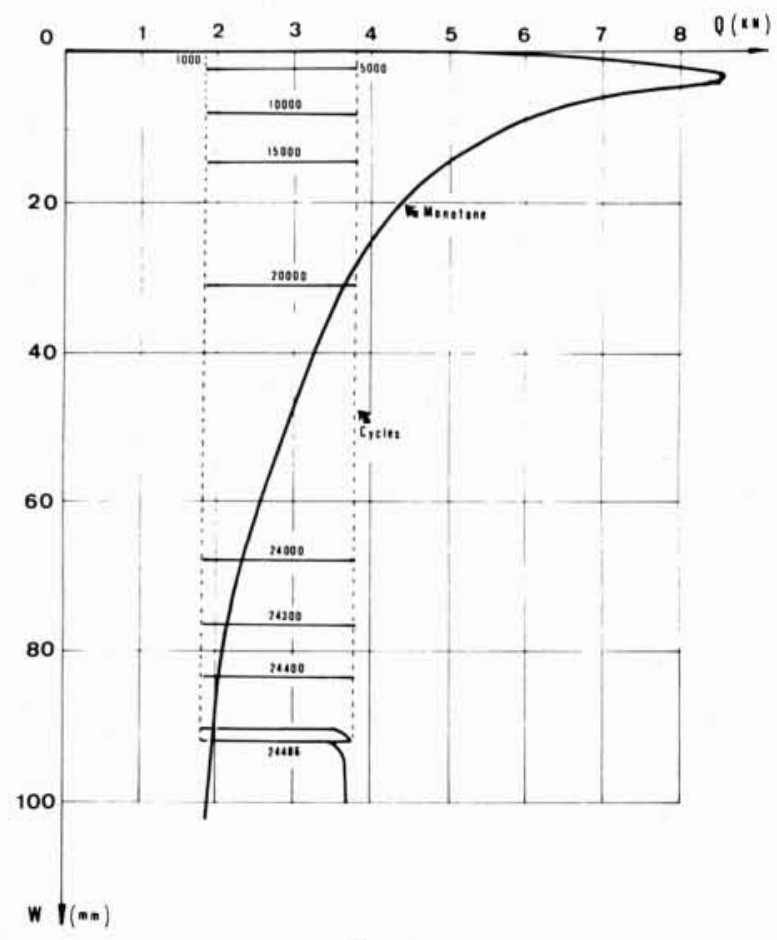

Figure 6. 
- d'une part, elle permet de séparer les problèmes (ce que ne permet pas l'étude globale), en mettant ainsi en évidence les mécanismes fondamentaux élémentaires qui sont déterminants dans le comportement;

- d'autre part, elle permet d'élaborer une modélisation réaliste de ces comportements élémentaires et de mettre au point des calculs prévisionnels.

Dans une première partie, nous exposons les données expérimentales de base qui mènent à l'identification de ces mécanismes élémentaires.

Dans une seconde partie nous abordons les moyens dont dispose le laboratoire pour simuler physiquement les problèmes d'interaction sol-structure.

Nous présentons enfin la manière dont les phénomènes majeurs qui interviennent au niveau de cette interaction peuvent être simulés numériquement sur ordinateur.

\section{1. - Données expérimentales de base}

Le but de la recherche expérimentale est d'abord de comprendre le comportement de l'ouvrage. Une première approche consiste à le visualiser qualitativement, ce qui est envisageable en laboratoire, mais ne l'est pas au niveau de l'ouvrage réel, surtout en milieu marin. Cette visualisation nous guide pour instrumenter ensuite des modèles de laboratoire qui quantifient ce comportement dans des conditions de sols et de chargement bien connues. C'est l'ensemble de ces deux points qui nous permet d'identifier ainsi les mécanismes élémentaires mis en jeu.

\subsection{Visualisation}

La visualisation du chargement de modèles d'ancrages ou de pieux donne des renseignements précieux, comme le montrent les exemples suivants.

Des essais d'arrachement de tirants horizontaux ont été réalisés dans un massif de sable de Fontainebleau par Plumelle [12] au CEBTP. Des bandes verticales de sable noir avaient été insérées dans le massif pour observer le domaine d'influence du tirant. Une coupe transversale du massif après arrachement montre que seule une mince zone de sol immédiatement autour du tirant, d'épaisseur 1 à $2 \mathrm{~mm}$ soit une dizaine de diamètres de grains, a été cisaillée (figure 1).

Des expériences analogues ont été par le passé effectuées par Robinsky et Morrisson [14] sur des pieux modèles. Le massif de sable comportait différents lits horizontaux de billes métalliques dont les auteurs pouvaient suivre les déplacements à partir de clichés aux rayons $\mathrm{X}$. Là encore on a mis en évidence que lors du fonçage du pieu, seule une zone très voisine du fût du pieu subit de grands déplacements (figure 2). Sous la pointe du pieu par contre on observe une zone très comprimée, grossièrement en forme de cône ou de bulbe (figure 3 ).

Une visualisation analogue a été réalisée à l'Ecole Centrale de Paris, dans un milieu analogique bidimensionnel formé de pailles compressibles et par Allersma [1] par photoélasticimétrie sur des billes de verre.

Globalement toutes ces expériences de visualisation confirment le comportement différent sous la pointe de l'ouvrage (zone comprimée soumise à des fortes contraintes) et en section courante (zone de grands cisaillements immédiatement au contact sol-inclusion). $\mathrm{Si}$ donc on cherche à quantifier le comportement, il faut instrumenter cet interface sol-pieu.

\subsection{Essais quantitatifs sur modèles}

Les expériences sur modèles ne constituent pas à proprement parler des modèles-réduits comme on peut en réaliser en hydraulique. Il s'agit simplement d'ouvrages de petite taille réalisés dans un environnement parfaitement connu (sable homogène de compacité donnée, argile consolidée uniformément) de façon à quantifier les comportements visualisés précédemment et à les relier à des caractéristiques mécaniques connues.

Un premier exemple est constitué par un essai d'arrachement que nous avons réalisé à I'I.M.G. [18] sur un pieu-modèle instrumenté de façon à mesurer à la fois la contrainte tangentielle et la contrainte normale en un point du fût lors du chargement. Le pieu était moulé dans du sable d'Hostun à forte compacité. La figure $4 a$ met nettement en évidence la très forte augmentation de la contrainte latérale (rapportée au poids des terres) lors du chargement: le coefficient $K=\sigma_{\text {lat }} / \gamma, z$ passe de 0,5 (poussée des terres au repos) à une valeur environ 7 fois plus forte à la rupture. Par contre la figure $4 b$ indique que le rapport $\tau / \sigma_{\text {lat }}$ atteint une valeur limite constante de l'ordre de 0,6 , qui correspond à la rugosité sol-pieu rugueux. La visualisation, puis cette expérience montrent donc clairement que le chargement ou l'arrachement d'un pieu ou d'un tirant doivent être appréhendés à partir de la notion de cisaillement localisé et de la variation de la contrainte normale latérale.

Ces considérations permettent de mieux situer et parfois de remettre en cause les règles couramment admises dans la pratique (valeur forfaitaire du coefficient $\mathrm{K})$.

D'autres expériences sur modèles, comme celles de Kérisel, Adam et Tcheng [9] ont permis de quantifier la résistance mobilisée sous la pointe en fonction de la profondeur d'ancrage du pieu (figure 5). Ces expériences ont conduit entre autres à regarder avec beaucoup de circonspection les schémas de calcul élaborés à partir d'une extrapolation des théories utilisés pour les fondations superficielles (coefficients $\mathrm{Nq}$ ).

Enfin, pour les ouvrages offshore, les expériences sur modèles nous permettent de quantifier de manière intéressante l'effet des sollicitations cycliques. La figure 6 montre la comparaison de deux essais d'arrachement de pieuxmodèles réalisés dans la cuve à sable de l'I.M.G., l'un sous sollicitation monotone, l'autre en appliquant au pieu des 

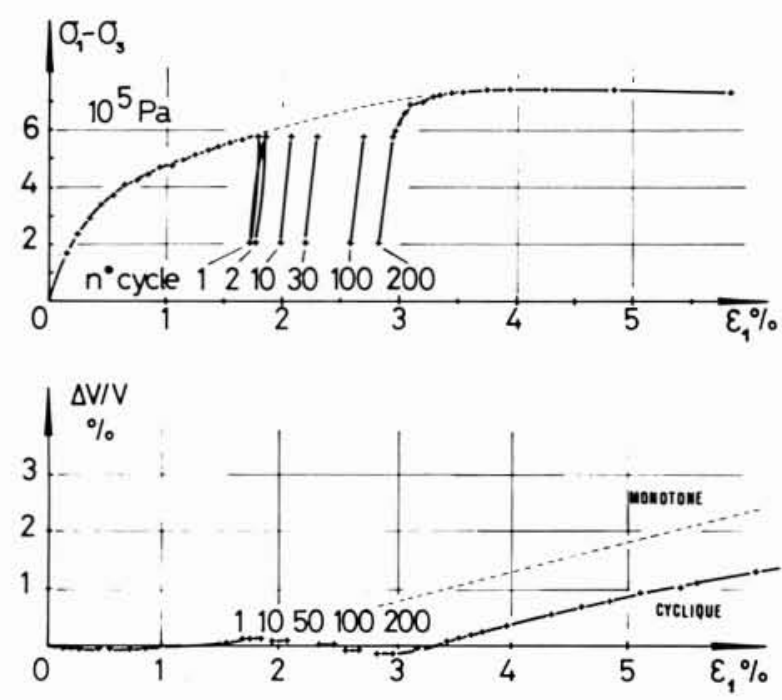

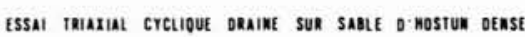

Figure 7. - daprès Thanopoulos [15].
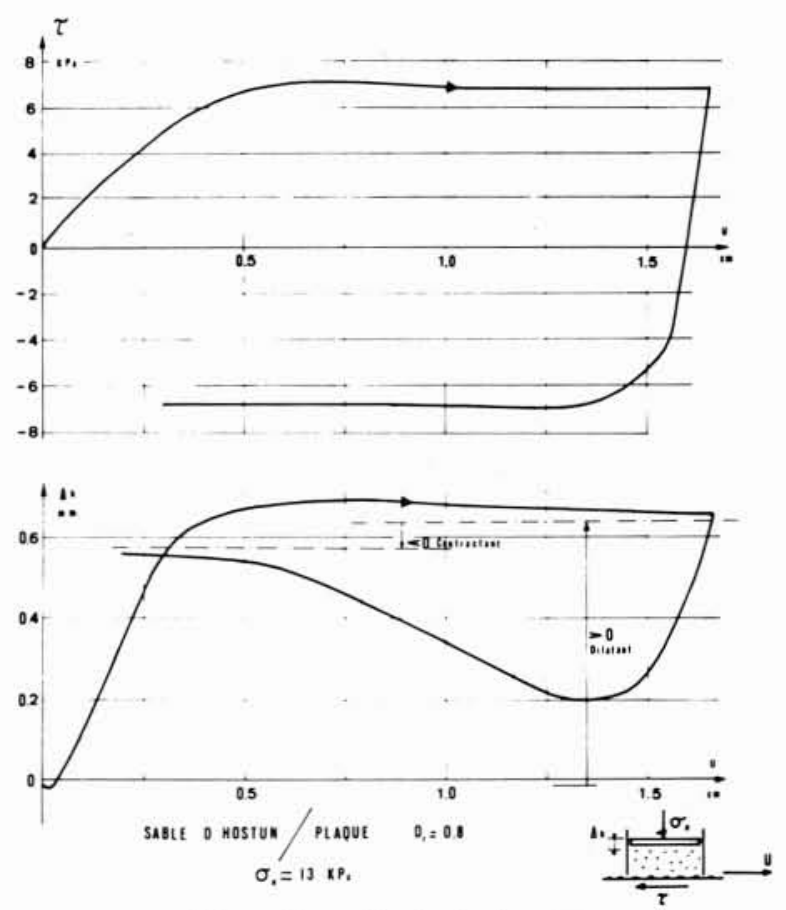

Figure 9. - d'après Boulon [6]

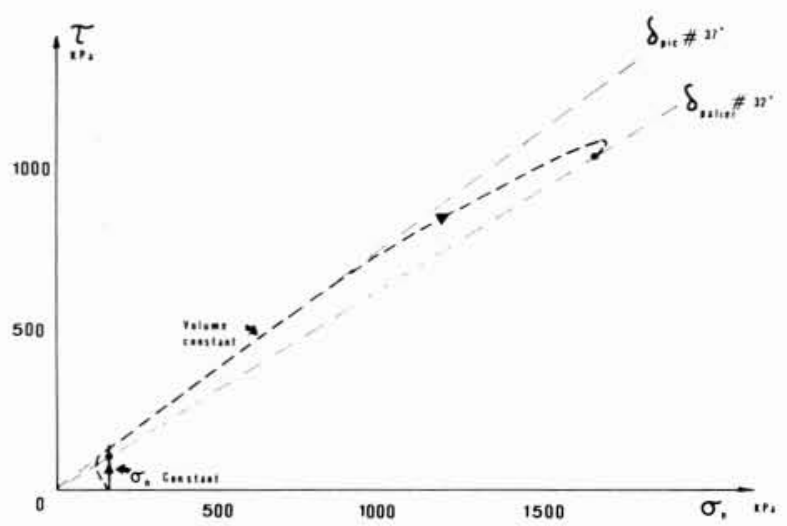

Figure 11. - d'après Boulon [6].

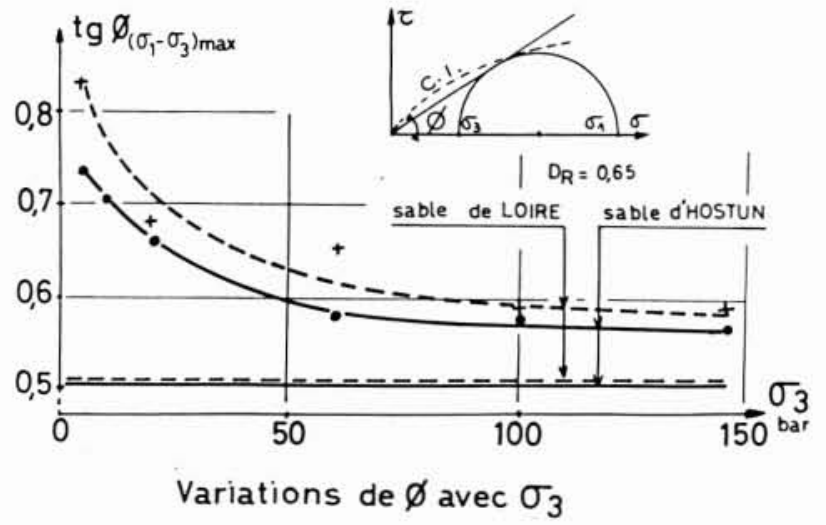

Figure 8. - d'après Le Long [11].
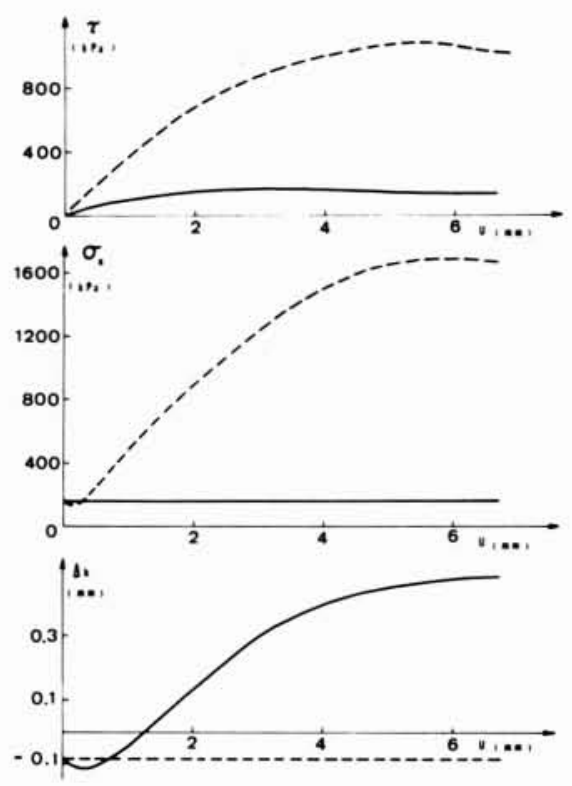

CISALLEMENT DIRECT SabLE hostun/PLAque rugueuse

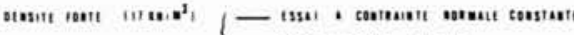

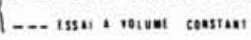

Figure 10. - d'après Boulon [6].

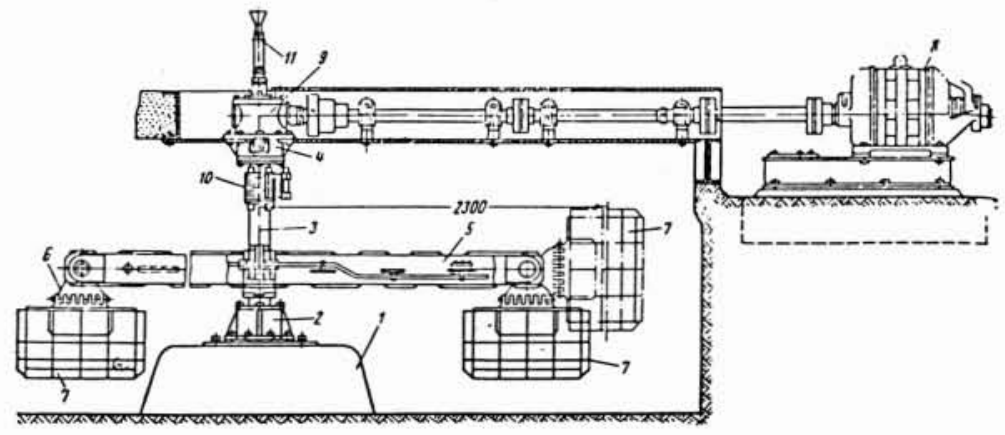

Figure 12. - Centrifugeuse soviétique permettant des accélérations de $250 \mathrm{~g}$. 
cycles de chargement autour d'une valeur moyenne égale environ au tiers de la charge de rupture statique et d'amplitude $\pm 12,5 \%$ de cette charge de rupture. On constate que le chargement cyclique bien qu'effectué dans un domaine éloigné de la rupture, produit néanmoins une accumulation de déplacements du pieu et une rupture après 25000 cycles [18]. Les cycles peuvent donc produire une dégradation importante du frottement sol-pieu, à prendre en compte au niveau du dimensionnement.

\subsection{Identification des mécanismes élémentaires}

Au vu des visualisations et essais sur pieux modèles en laboratoire présentés ci-dessus, il est possible de dégager les phénomènes élémentaires déterminants, qu'on peut isoler et reproduire en laboratoire, et qui conditionnent l'ensemble de la réponse de la fondation. Il s'agit principalement des comportements du sol "dans sa masse " et des interfaces sol-structures.

Le comportement du sol "dans sa masse " est identifiable grâce à des essais triaxiaux de révolution classiques, tandis que la boîte de cisaillement direct apparait comme le meilleur outil actuel d'étude des interfaces. Bien entendu ces essais ne sont pas exempts de défauts, quant à l'homogénéité notamment. La notion de chemin, en contraintes par exemple, est essentielle lors de l'interprétation d'essais mécaniques sur les sols.

La figure 7 présente un essai triaxial drainé sur sable dense. On remarquera le comportement essentiellement dilatant sur chemin monotone, et au contraire contractant sur chemin cyclique. Cette lente accumulation des déformations irréversibles est à rapprocher de la dégradation du frottement latéral le long des pieux sollicités cycliquement.

Un autre aspect important du comportement des sols mesuré sur chemin triaxial est constitué par la diminution importante de l'angle de frottement entre faible et fort niveau de contraintes (cf. Fig. 8). Cette propriété est en relation directe avec les efforts de pointe limités observables lors de la mise en place d'un pieu par fonçage ou battage.

Les comportements d'interface (cisaillement direct sol-plaque rugueux) présentent des caractéristiques très semblables aux comportements du sol "dans la masse". Ainsi (cf. Fig. 9), un cisaillement monotone à contrainte normale contante sur sable dense fait naitre une dilatance importante, tandis qu'un changement de sens de sollicitation entraîne une contractance "tangente ". Mais l'idée essentielle permettant de saisir le comportement d'interface (qui conditionne le frottement sol-pieu) est l'énorme différence entre un chemin à contrainte normale constante et un chemin à volume constant (cf. Fig. 10) en ce qui concerne la contrainte de cisaillement $(\tau)$, la contrainte normale $\left(\sigma_{n}\right)$ et la quantité conventionnellement appelée variation de volume $(\Delta h)$. Cette différence, qui s'explique par le couplage entre phénomènes tangentiels et normaux à l'intérieur d'une bande de cisaillement, peut conduire au cours d'un essai à volume constant, selon le type de matériau, à des augmentations importantes de contrainte normale (sables quartzeux par exemple), qui elle-mêmes entraînent de forts accroissements de contrainte normale constante, ou au contraire à des diminutions considérables de contraintes dans le cas de matériaux fragiles (sables calcaires par exemple). Les chemins à volume constant et à contrainte normale constante peuvent être considérés comme des chemins réels observés lors de la mobilisation du frottement latéral le long des pieux; la figure 11 qui complète la précédente, précise l'écart entre les frottements mobilisés (rapport 1 à 10 ) et montre clairement qu'un essai classique de cisaillement direct à contrainte normale constante ne peut rien donner de plus qu'un angle de frottement, ce qui est insuffisant pour prévoir le frottement latéral.

\section{2. - Simulation physique}

Les expériences dont nous avons rendu compte dans la première partie sont intéressantes pour la compréhension et l'identification des phénomènes mis en jeu. Mais à part cet intérêt académique, elles se heurtent à la critique du praticien en ce sens qu'elles ne simulent pas les conditions réelles (dimension et profondeur) de l'ouvrage. En ce qui concerne les ouvrages à la mer, cette critique s'adresse aussi bien aux essais de pieux in-situ réalisés à terre (diamètre 30 à $40 \mathrm{~cm}$, profondeur 20 à $30 \mathrm{~m}$ ) qui ne constituent que des essais en semi-grandeur.

\subsection{Similitude et centrifugeuse}

Si l'on souhaite donc expérimenter en laboratoire un modèle de fondation ou d'ancrage à l'échelle $1 / N$, en utilisant le sol réel et si on désire obtenir le niveau de contraintes auquel sera réellement soumis l'ouvrage in-situ, les lois de similitude en mécanique des sols Weber [16] - impliquent que l'on impose au modèle une gravité $\mathrm{N}$ fois plus forte. Ceci peut être réalisé par des modèles à gradient hydraulique, comme celui de Zelikson [17], mais surtout en effectuant des expérimentations en centrifugeuse. La figure 12 montre le schéma d'une telle centrifugeuse permettant d'appliquer à la nacelle contenant le modèle à expérimenter des accélérations de 100 à $200 \mathrm{~g}$.

L'avantage de la centrifugeuse est de pouvoir étudier le comportement global d'ouvrages complexes: plateforme fondée sur groupes de pieux, digues, etc. L'ensemble peut être instrumenté et les expériences suivies par caméra. On peut essayer de vérifier le comportement global dans un cas de chargement précis (cycles en particulier). En France, un tel appareillage existe au CESTA à Bordeaux et une centrifugeuse à usage géotechnique est en fin de construction au LCPC de Nantes. 


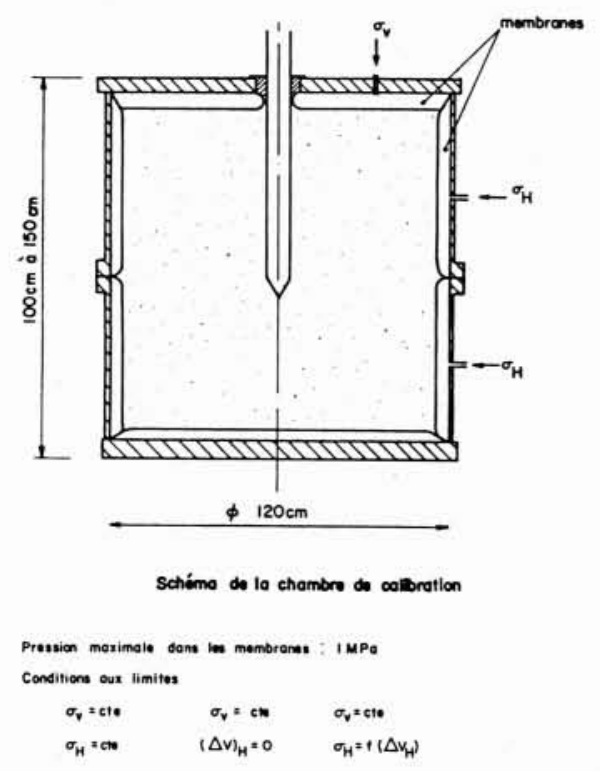

Figure 13. - Schéma de la chambre de calibration.

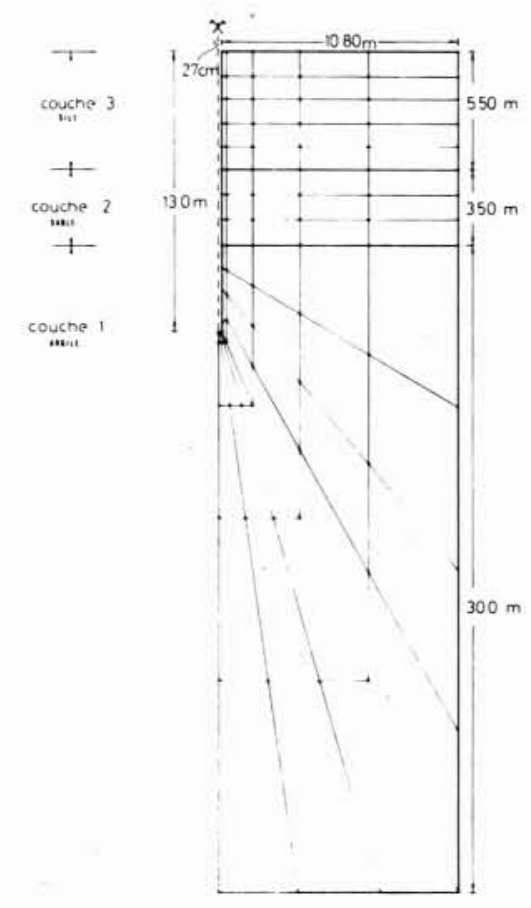

Figure 15

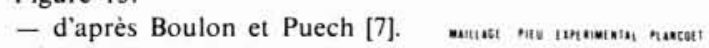

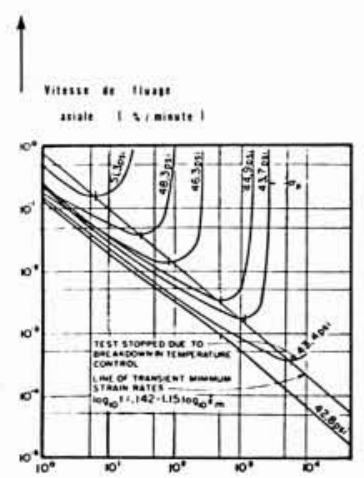

t..., $\overrightarrow{\text { sinten }}$,

fluage - oue argite notmalement cansolices, astes fink (14)

$\sigma_{1}: 295,354 \mathrm{tp}$

$\sigma_{\text {s }}: 517 \mathrm{pt}$

(a)

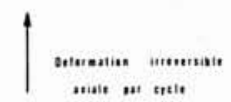

niate an trtis

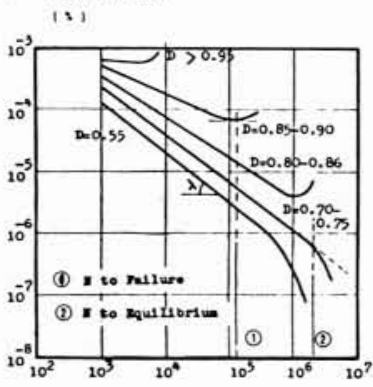

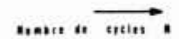

Oetormations speligues irrerersibiss

Cone agile

C ates tashine III) (b)

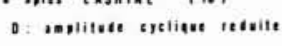

analogie fluage / DEFormations cycliques irreversibles

Figure 17. - d'après Finn [8] et Lashine [10].

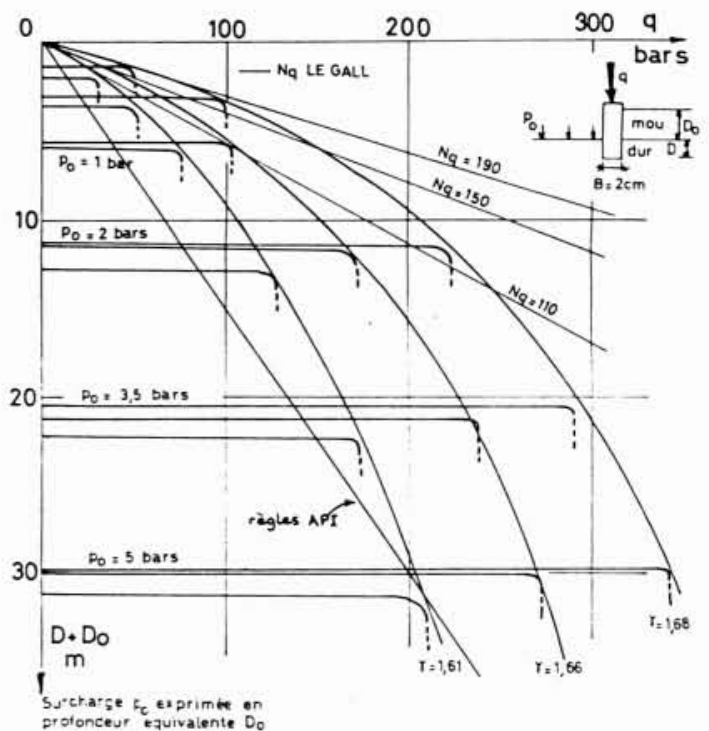

Figure 14. - Pénétrations en milieu surchargé - Effort de pointe - (Essais Dessaint).

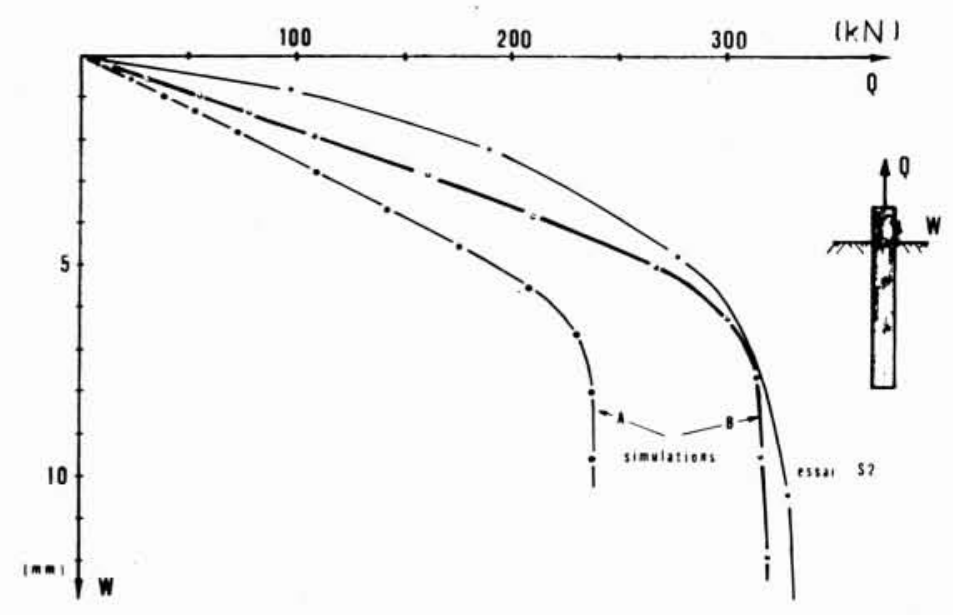

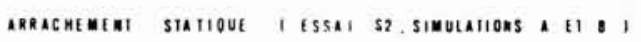

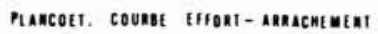

Figure 16. - d'après Boulon et Puech [7].
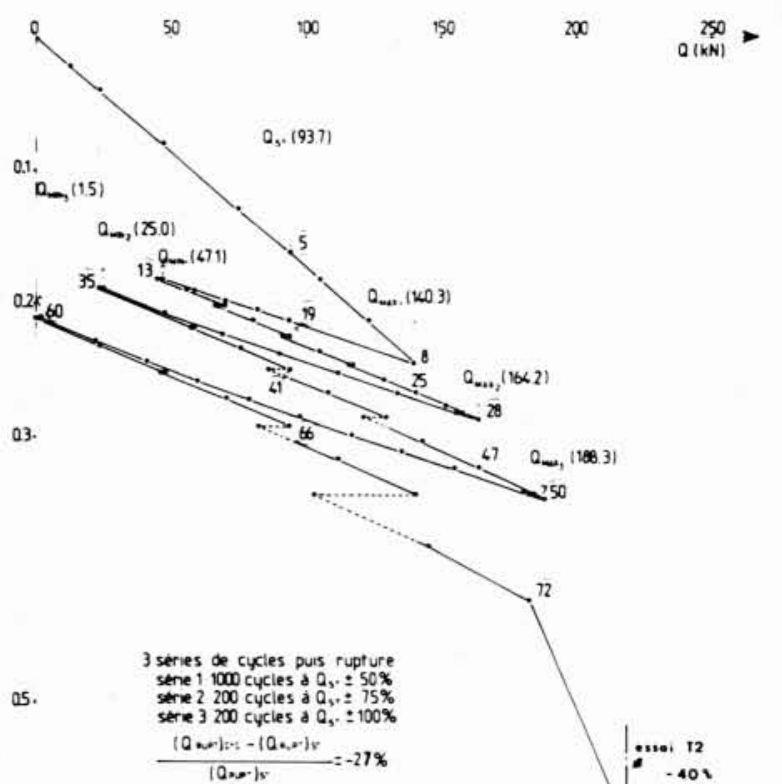

06 .

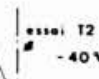

07. WI $/ \mathrm{cm}$ !

v heu of plancoet

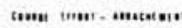




\subsection{Chambre de calibration}

Un modèle intermédiaire entre le modèle simple et la cenrifugeuse a été développé pour simuler de façon correcte le niveau de contrainte réellement appliqué in situ. Il s'agit de la chambre de calibration. Celle-ci est constituée d'une cuve rigide de $1 \mathrm{~m}$ à $1,5 \mathrm{~m}$ de diamètre et de hauteur environ $1,5 \mathrm{~m}$ dans laquelle on vient placer le sol à une compacité donnée. Les parois verticales et latérales de la cuve comportent des membranes, qui permettent d'appliquer au sol des conditions aux limites imposées du type suivant par exemple :

- en vertical : une pression $\sigma_{i}=$ constante simulant l'effet de la profondeur;

- en horizontal : une pression $\sigma_{h}=$ cte imposant au matériau une histoire particulière par son coefficient $K o=\sigma_{h} / \sigma_{i}$, ou une déformation $\Delta \varepsilon_{h}=0$ qui impose des conditions de type oedométrique.

La figure 13 montre un schèma de la chambre réalisée à l'I.M.G. A l'intérieur de cet échantillon de sol, on peut effectuer des essais de pénétration ou de chargement de pieux-modèles instrumentés. On peut donc "calibrer " la réponse de l'appareil (en résistance de pointe et frottement latéral) en fonction de la profondeur simulée, de la compacité du sol, de son histoire (Ko, OCR), du mode de chargement.

Le dispositif expérimental permet également d'étudier des pieux ou ancrages de plus gros diamètre traversant la cuve de part en part. On simule alors le comportement réel d'un tronçon de l'ouvrage. La chambre de calibration constitue donc un outil de simulation physique nous permettant d'effectuer la liaison entre les comportements élémentaires et le comportement de l'ensemble de la fondation.

La figure 14 indique à titre d'exemple des résultats d'essais en petite chambre de calibration obtenus à l'IMG par Dessaint et concernant la résistance de pointe de petits pieux. Ces résultats mettent en évidence le peu de confiance que l'on peut accorder aux relations du type $q=\gamma D N q$ ainsi qu'à la grosse approximation faite par les règles API $(N q=40)$.

\section{3. - Simulation numérique}

La base de la simulation numérique est constituée par la méthode des éléments finis (technique de discrétisation de l'espace, du temps, des propriétés mécaniques, et des équations d'équilibre), s'appuyant elle même sur le principe du travail virtuel. Ce type de méthodes nécessitait il y a quelques années de très gros ordinateurs, mais l'évolution de l'électronique et de l'informatique sont telles qu'on commence à traiter ces problèmes à partir de micro-ordinateurs.

La première phase du traitement consiste en la discrétisation spatiale du problème (maillage) dont la figure 15 donne une idée en ce qui concerne le calcul relatif au pieu expérimental de Plancoet, décrit sur cette même figure, et financé par l'Institut Français du Pétrole. La souplesse de la discrétisation doit permettre d'adapter les "éléments " à la forme des diverses couches de sol.

La seconde phase consiste en l'identification d'une loi rhéologique " en volume" pour le sol, à partir d'essais triaxiaux, et d'une loi d'interface pour le contact solstructure à partir d'essais de cisaillement direct. Les grands types de lois actuellement utilisées sont les lois élasto-plastiques, basées sur des modèles à priori, et les lois à tendance plutôt phénoménologiques, improprement appelées lois incrémentales. Le passage des essais mécaniques in situ à la rhéologie utilisable en simulation numérique reste encore très incertain, et principalement basé sur des corrélations.

Le pressiomètre et son adaptation marine mise au point par l'Institut Français du Pétrole sont à cet égard très intéressants.

La simulation d'une sollicitation monotone en tension est présentée à la figure 16 avec une loi de type incrémental. Les données mécaniques brutes conduisent à la courbe A qui montre un certain écart avec la réalité expérimentale de l'essai in situ S2. Ceci est dû au fait que le calcul simule encore très mal l'histoire de l'installation d'un pieu foncé ou battu. Les divers auteurs «forcent" artificiellement les propriétés mécaniques du sol situé au voisinage du pieu pour rendre compte de ce phénomène (courbe B).

La simulation d'une sollicitation cyclique en tension à grand nombre de cycles exige tout d'abord une formulation particulière des propriétés mécaniques sous cycles. Le pseudo-fluage cyclique, dans lequel le nombre de cycles tient lieu de temps fictif est un outil intéressant, lorsqu'on examine (cf. fig. 17) les analogies entre fluage réel et déformations irréversibles cycliques. Cette formulation permet également une économie importante de temps de calcul (cf. G. Bouckovalas et al. [3]). L'application de cette technique nous a permis de décrire 1400 cycles de chargement à partir de 73 increments de calcul seulement, dont 12 de pseudo-fluage cyclique (cf. fig. 18) en conduisant à une réduction du frottement latéral après cycles de $27 \%$ (contre $40 \%$ observé expérimentalement). La validité de tels calculs repose sur de nombreuses comparaisons ponctuelles calcul expérience, dont par exemple les déplacements en tête de pieu (cf. fig. 19), d'autres comparaisons sont présentées par Puech et al. [5]

La méthode des éléments finis permet également de simuler, pour les matériaux peu perméables, l'interaction sol-fluide interstitiel, par discrétisation des bilans de volume locaux, ce qui conduit au champ de pressions interstitielles (cf. Fig. 20). Il est notable de remarquer que l'interaction sol-pieu est fort différente, selon qu'on adopte l'hypothèse de sol draîné ou non-draîné au voisinage du pieu. 


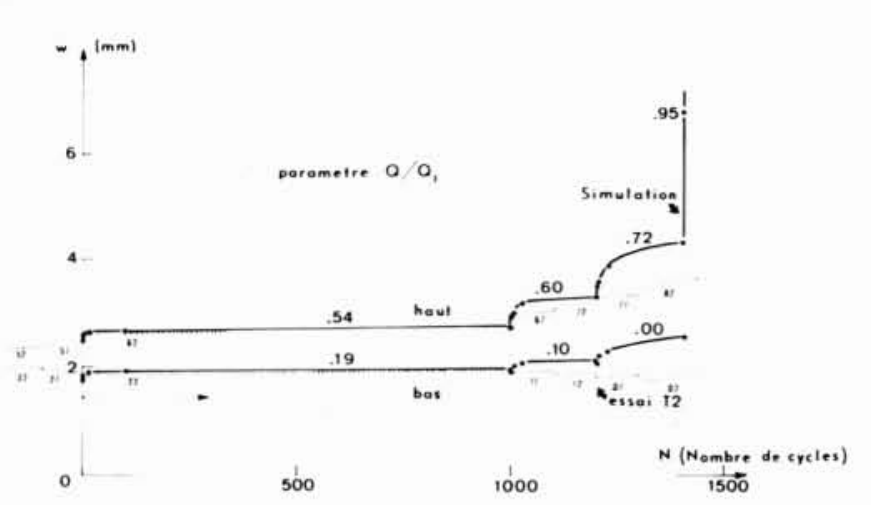

PLANCOET SIMULATION ESSAI tEMPETE DEPLACEMENTS EN TEIE haUt ET BAS DE CYCLES

Figure 19. - daprès Boulon et al. [7].

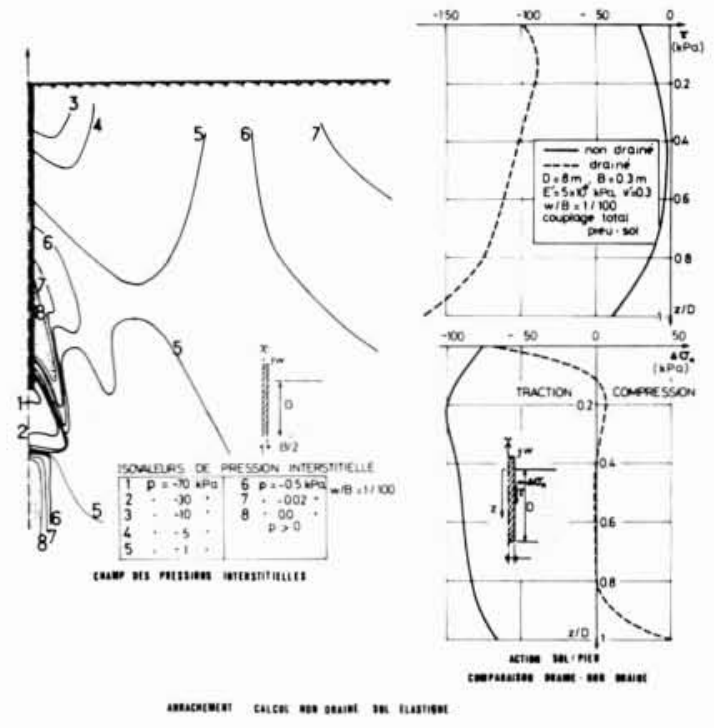

Figure 20. - d'après Boulon et al. [4].

\section{Conclusion}

Le progrès des connaissances sur le comportement des fondations offshore a un grand besoin simultané d'essais in situ bien instrumentés et d'essais de laboratoire sur modèles ou destinés à formuler les mécanismes élementaires intervenant dans le sol ou au contact sol-structure. Parallèlement, l'auscultation en place des ouvrages existants est un moyen d'investigation à ne pas négliger. Au-delà d'une certaine complexité des modèles de calcul, se pose la question d'existence de modèles simplifiés fiables d'aide à la conception.

L'apport de la recherche en laboratoire dans ce domaine peut justement consister en la sélection d'un petit nombre de paramètres majeurs gouvernant la compressibilité des sols et le frottement sol-structure d'une part, et en l'élaboration des modèles simples qui en découlent d'autre part. Ne nous cachons pas que la liaison entre ces paramètres et les essais de reconnaissance in situ reste un problème largement ouvert.

\section{Bibliographie}

[1] Allersma H.G.B. - Photoelastic investigation of the stress distribution during penetration. Second European Symposium on penetration testing, vol. II, pp. 411-418, Amsterdam, May 1982.

[2] Bellotti R; Bizzi G., Ghionna V., Jamiolkowski M., MarchettI S., PASQualini E. - Preliminary calibration tests of electrical cone and flat dilatometer in sand. VII European Conf. on Soil Mechanics and Foundation Engineering, vol. 2, pp. 195-200, Brighton, September 1979.

[3] Bouckovalas G., Whitman R.V., allen marr W. Permanent displacement of sand with cyclic loading. ASCE, Journ. of Geotechnical Engineering, vol. 110, $\mathrm{n}^{\circ} 11$, pp. 1606-1623, november 1984.

[4] Boulon M., Darve F., Flavigny E., Forgue M. - Méthode de prévision des pressions interstitielles. Revue Française de Géotechnique, n 9, pp. 27-40, décembre 1979.

[5] Puech A., Boulon M., Meimon Y. - Behavior of tension piles : field data and numerical modelling. Second Internatio- nal Conference on numerical methods in offshore piling. Austin, USA, April 1982

[6] BouLon M. - Une approche simplifiée du frottement latéral le long des pieux et tirants d'ancrage. Actes $d u$ Colloque National Génie Civil et Recherche, Tome II, pp. 231-249, Paris, juin 1983.

[7] Boulon M., PUeCH A. - Simulation numérique du comportement des pieux sous chargement axial cyclique. Rerue Française de Géotechnique, n²6, pp. 7-20, juin 1984.

[8] FINN W.D.L., SHEAD D. - Creep and creep rupture of an undisturbed sensitive clay Proc. of the VIII Int. Conf. on Soil Mechanics and Foundation Engineering, pp. 135-142, Moscou, 1973.

[9] Kerisel, AdAM, TCHeng. - Fondations profondes. AITBTP, $\mathrm{n}^{\circ} 179,1962$.

[10] LASHINE A.K.F. - Deformation characteristics of a silty clay under repeated loading. Proc. of the VIII Int. Conf. on Soil Mechanics and Foundation Engineering, pp. 237-244, Moscou, 1973.

[11] LE LONG. - Contribution à l'étude du comportement des sables sous forte pression. Thèse de Docteur-Ingénieur, Université de Grenoble, 1968.

[12] Plumelle C. - Etude expérimentale du comportement des tirants d'ancrage. Thèse de Docteur-Ingénieur, Université de Paris VI, décembre 1979.

[13] Puech A., Foray P., Boulon M., Desrues J. - Calcul des pieux à l'arrachement à partir d'un modèle numérique en contraintes effectives. VII European Conference on Soil Mechanics and Foundation Engineering, vol. 1, pp. 227-232, Brighton, September 1979.

[14] RoBinSKY E.I., Morrison C.F. - Sand displacement and compaction around model friction piles. Canadian Geotechnical Journal, vol. 1, N $\mathrm{N}^{\circ}$ 2, March 1964.

[15] Thanopoulos I. - Contribution à létude du comportement cyclique des milieux pulvérulents. Thèse de Docteur-Ingénieur, Université de Grenoble, 1981.

[16] WEBER. - La similitude en mécanique des sols. Eyrolles, Paris, 1970.

[17] ZeLikson A. - Geotechnical models using the hydraulic gradient similarity method. Géotechnique, vol. 19, $\mathrm{n}^{\circ} 4,1969$.

[18] Calcul des pieux sous sollicitations cycliques. Rapport de Recherche I.M.G.-I.F.P., novembre 1979. 